\title{
Carbon-Encapsulated Magnetic Nanoparticles Based on Fe, Mn, and Cr for Spintronics Applications
}

\author{
J. Szczytho ${ }^{a}, \mathrm{P}$. Osewski ${ }^{a}$, M. BystrzejeWski ${ }^{b}$, \\ J. Borysiuk ${ }^{c}$, A. Grabias ${ }^{c}$, A. $\mathrm{HuCZKo}^{b}, \mathrm{H} . \mathrm{LANGE}^{b}$, \\ A. MAJHOFER ${ }^{a}$ AND A. TWARDOWSKI ${ }^{a}$ \\ ${ }^{a}$ Institute of Experimental Physics, Faculty of Physics, Warsaw University \\ Hoża 69, 00-681 Warsaw, Poland \\ ${ }^{b}$ Department of Chemistry, Warsaw University \\ Pasteura 1, 02-093 Warsaw, Poland \\ ${ }^{c}$ Institute of Electronic Materials Technology \\ Wólczyńska 133, 01-919 Warsaw, Poland
}

\begin{abstract}
We succeeded in the arc-plasma synthesis of carbon-encapsulated Fe, $\mathrm{Cr}$, and Mn-based nanoparticles. The transmission electron microscopy, Mössbauer spectra (of iron) and SQUID magnetometry results demonstrate that the products of the synthesis contain metals and its carbides. The nanoparticles show ferromagnetic or superparamagnetic behavior at high temperatures, which is demanded for nano-spintronics applications.
\end{abstract}

PACS numbers: 75.20.-g, 75.75.+a, 75.20.En, 75.30.Cr, 75.50.Bb

\section{Introduction}

Carbon-based nanostructured materials are the promising candidates for future applications in novel electronics. Unique flexibility in $s p$-hybridization of carbon valence orbitals results in different stable carbon allotropic forms, affinity for bonding with other atoms and gives nearly ten million different chemical compounds, with the potential use in miniaturized electronics. Carbon nanotube transistors and logic gates, conducting carbon-based polymers have been already demonstrated [1-7].

Graphite is one of the candidates for future electronics - single graphene layers form two-dimensional materials and show many interesting physical phenomena in the presence of magnetic field, like half integer quantum Hall effect, 
electron-hole degeneracy, and vanishing carrier mass near the point of charge neutrality. Intercalation of graphite with various elements yields charge-transfer compounds that may be considered as an analog of semiconductor doping $[8,9]$.

In this communication we propose to integrate ferromagnetic nanomaterials with several graphene layers in so-called carbon-encapsulated magnetic nanoparticles (CEMNPs) [10-16]. Such hybrid structures possess interesting chemical and physical properties. The carbon shell protects the encapsulated crystallites from the oxidation and further agglomeration. Moreover, the magnetic field from the magnetic core should influence on graphite band electrons [9].

\section{Synthesis of nanomaterials}

The synthesis was carried out in an arc discharge chamber in helium atmosphere. The discharge current and voltage was between $70 \mathrm{~A}$ and $25 \mathrm{~V}$, respectively. The graphite anodes $(6 \mathrm{~mm}$ in diameter, $10 \mathrm{~cm}$ in length) were drilled (3 $\mathrm{mm}$ in diameter, $3 \mathrm{~cm}$ in length) and filled with a mixture of pure $\mathrm{Fe}$ or $\mathrm{Cr}$ or Mn (depending on the process) and graphite powder. The as-prepared anodes contained $12-14 \%$ at. of metal. The pure graphite rods were used as cathodes. The as-prepared electrodes were arc sublimated. The resulted soot was collected from the walls of the reactor. All products were subjected to the purification procedure (to remove non-encapsulated metals). A sample of soot was put into a flask, boiled with $\mathrm{HCl}$ for $24 \mathrm{~h}$, then washed with plenty of water and ethanol, and finally dried in air at $380 \mathrm{~K}[16]$.

\section{The experimental results and discussions}

\subsection{Transmission electron microscopy}

Morphology of nanosized Mn, Fe, and Cr-based CEMNPs was studied by the transmission electron microscopy (TEM). The core of each nanocapsule consists of magnetic metal or its carbides. Such a core is surrounded by several graphite layers (as in the case of $\mathrm{Fe}$ and $\mathrm{Mn}$ ) or by the amorphous carbon $(\mathrm{Cr})$. The synthesis results in a mixture of the nanoparticles with different sizes, from several nm

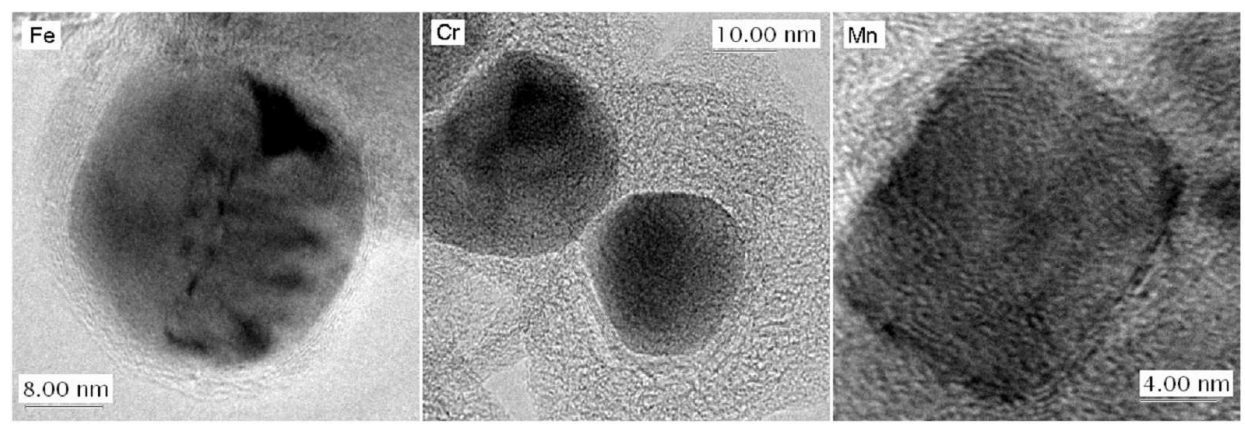

Fig. 1. Morphology of nanosized Mn, Fe, and Cr-based CEMNPs. 
to over $100 \mathrm{~nm}$. Typical morphology of obtained particles is presented in Fig. 1. Usually the nanoparticles are attached to each other forming a kind of large agglomerates. This is due to the high surface energy of the nanoparticles. Among the nanoparticles there is also an amorphous carbon and soot.

Selected area electron diffraction provides a detailed piece of information about the phase composition. The peaks corresponding to pure metallic phases and metal-carbides were found in the diffraction patterns. The more quantitative results of the core composition were obtained by the Mössbauer spectroscopy.

\subsection{Mössbauer spectroscopy}

The Mössbauer spectra were measured in transmission geometry at room temperature. A constant-acceleration spectrometer with a $25 \mathrm{mCi}{ }^{57} \mathrm{Co}-\mathrm{in}-\mathrm{Rh}$ source was used. The Mössbauer measurements allowed the identification of iron-containing phases in Fe-based nanoparticles. The relative fractions of the phases were calculated as a ratio of the area of the relevant subspectrum to the total spectral area, assuming similar Debye-Waller factors for each phase. Isomer shifts are given with respect to $\alpha$-Fe foil.

The fitting of the Mössbauer spectra allowed the evaluation of the relative atomic fractions of iron as the magnetic or paramagnetic phases (Fig. 2). The quantitative analysis evidences the difference in the relative contents of the magnetic phases observed in the Fe-based samples studied. A significant contribution of the magnetic carbide $\mathrm{Fe}_{3} \mathrm{C}$ phase is observed (43\%). The relative fractions of the magnetic $\alpha$-Fe and paramagnetic $\gamma$-Fe phases are $36 \%$ and $21 \%$, respectively. The rest of $\mathrm{Fe}$ is in different phases of $\mathrm{Fe}-\mathrm{C}$ mixture, mostly in an austenite $\gamma$ - $\mathrm{Fe}(\mathrm{C})$.

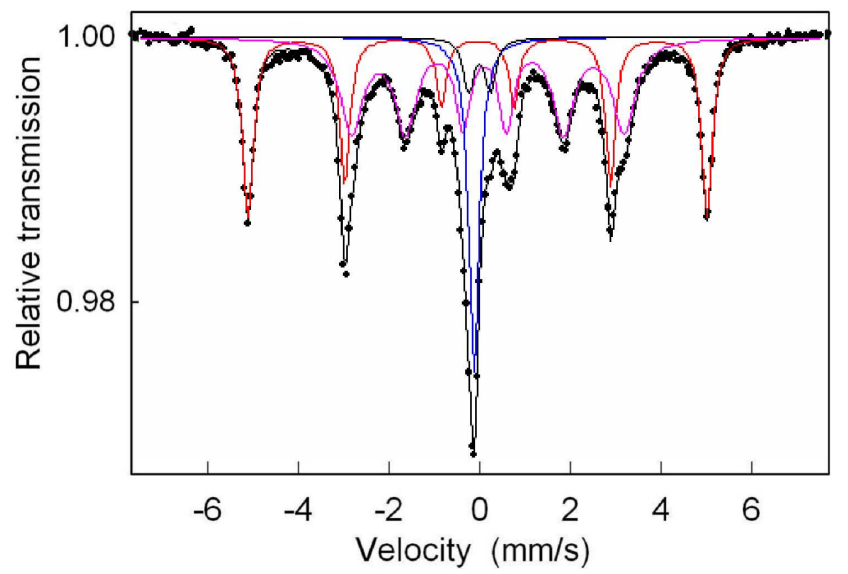

Fig. 2. Mössbauer measurements of iron-containing phases in Fe-based nanoparticles. Experimental points are marked by circles. The fitting curve consists of four phases $\alpha$-Fe, $\gamma$-Fe, $\gamma$-Fe(C) (austenite), and $\mathrm{Fe}_{3} \mathrm{C}$. 


\subsection{Magnetic measurements}

Magnetic properties of encapsulated Mn, Fe, and Cr (and their carbides) were studied by means of SQUID magnetometry in the temperature range of 10.0-300.0 K and magnetic fields 0.0-6.0 T. Diamagnetic contributions to the signal (resulting from amorphous carbon and graphite powder) were subtracted in order to obtain saturation at high magnetic fields. The CEMNPs showed ferromagnetic and superparamagnetic behavior at high temperatures, similar for $\mathrm{Mn}, \mathrm{Fe}$, and $\mathrm{Cr}$, while at low temperatures different behavior was observed, which depended on composition of magnetic cores (Fig. 3). If the nanocapsules contain pure metallic manganese or chromium one should expect antiferromagnetic behavior. Indeed, the antiferromagnetism manifests at low temperature, where the magnetization attains saturation at higher magnetic fields. However even at $10.0 \mathrm{~K}$ the dominant signal comes from ferromagnetic interaction, which means that $\mathrm{Mn}, \mathrm{Fe}$, and Cr-based CEMNPs contain ferromagnetic particles.
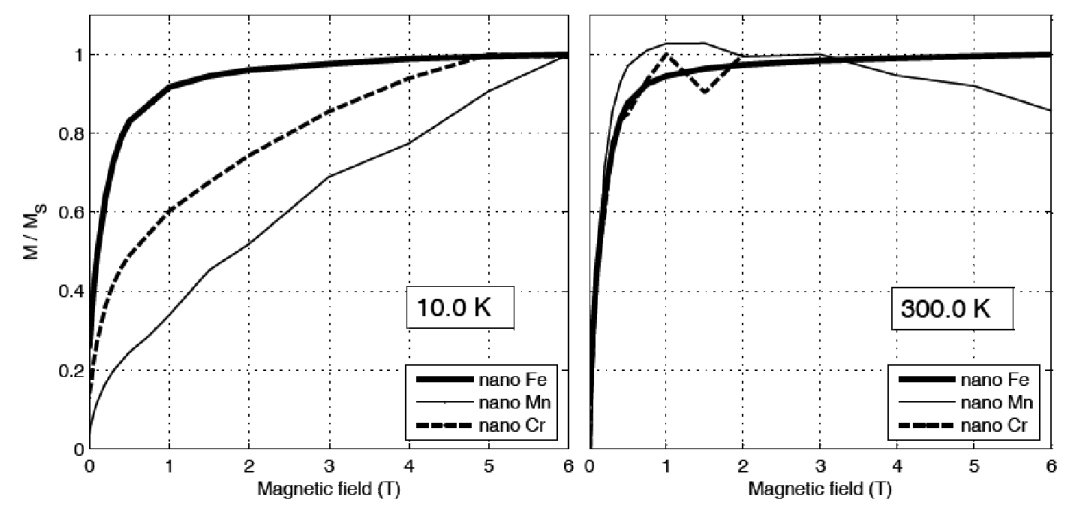

Fig. 3. Magnetization of encapsulated $\mathrm{Mn}, \mathrm{Fe}$, and $\mathrm{Cr}$ (and their carbides) as a function of magnetic field at $10.0 \mathrm{~K}$ and $300.0 \mathrm{~K}$. Diamagnetic contributions were subtracted. Magnetization $M$ is normalized to the value of saturation magnetization $M_{\mathrm{S}}$ which is (in emu/g units) at $10 \mathrm{~K}$ for Fe $M_{\mathrm{S}}=60, \mathrm{Cr} M_{\mathrm{S}}=1.0, \mathrm{Mn} M_{\mathrm{S}}=1.2$ and at $300 \mathrm{~K}$ for Fe $M_{\mathrm{S}}=53$, Cr $M_{\mathrm{S}}=0.4, \mathrm{Mn} M_{\mathrm{S}}=0.15$.

This ferromagnetism is probably caused by some metal-carbide compounds, similarly to the Fe-carbides, whose presence was detected by means of the Mössbauer spectroscopy (Fig. 2). Magnetic properties of Mn-carbide were not investigated yet, however it was mentioned that it is ferromagnetic [17]. Chromium carbides like $\mathrm{Cr}_{3} \mathrm{C}_{2}, \mathrm{Cr}_{23} \mathrm{C}_{6}, \mathrm{Cr}_{7} \mathrm{C}_{3}$ are known (from the literature of structure investigations), but there is no information about their magnetic properties. If samples contain also metal oxides one can expect antiferromagnetism of $\mathrm{MnO}$ and ferromagnetism of $\mathrm{CrO}_{2}$. However, since the synthesis of $\mathrm{Fe}, \mathrm{Mn}$, and $\mathrm{Cr}$ nanocapsules is similar, and since there was no evidence of any Fe-oxides from 
the Mössbauer spectroscopy (Fig. 2), one should rather exclude the presence of metal-oxides in our samples. Therefore, the ferromagnetic properties of investigated CEMNPs are due to the ferromagnetic properties of metal-carbides.

At higher temperatures, the magnetization saturates at $0.5 \mathrm{~T}$ (Fig. 3). The value of observed coercivity for each sample is small and comparable to the coercivity of the superconducting coil of the SQUID magnetometer. Therefore, it is difficult to judge if CEMNPs are ferro- or superparamagnetic and some further investigations are needed.

For spintronics devices working at room temperatures the origin of magnetism of nanoparticles is irrelevant [18]. However the value of saturation magnetization of iron-based nanoparticles is almost 60 times larger than the one of manganese or chromium (Fig. 3 caption). Therefore, the iron-based nanoparticles are more preferable for future spintronics devices.

\section{Conclusions}

We succeeded in the arc-plasma synthesis of $\mathrm{Fe}, \mathrm{Cr}$, and Mn-based nanoparticles. The plasma synthesis in carbon arc provides also carbides and therefore the obtained material is non-homogeneous. The nanoparticles show ferromagnetic or superparamagnetic behavior at high temperatures, which is demanded for nanospintronics applications. The next step toward the practical applications will focus on the removing the amorphous carbon and the separation of the magnetic phases via sedimentation route.

\section{Acknowledgments}

This work was supported by the Ministry of Science and Education through the Department of Chemistry, Warsaw University under grants No. N204 096 31/2160 and 3 T08D 012 28. Author (M.B.) thanks the Foundation for Polish Science (FNP) for the financial support. The use of TEM facilities at the Faculty of the Materials Science and Engineering of Warsaw University of Technology is kindly appreciated.

\section{References}

[1] X.X. Zhang, G.H. Wen, S. Huang, D. Liming, G. Ruiping, Z.L. Wang, J. Magn. Magn. Mater. 231, 9 (2001).

[2] R.H. Kodama, J. Magn. Magn. Mater. 200, 359 (1999).

[3] S.C. Tang, J. Qiu, J.F. Harris, Q.J. Fu, N. Zhang, Chem. Phys. Lett. 322, 553 (2000).

[4] H. Song, X. Chen, Chem. Phys. Lett. 374, 400 (2003).

[5] Z.H. Wang, Z.D. Zhang, C.J. Choi, B.K. Kim, J. Alloys Comp. 361, 289 (2003).

[6] S. Mornet, S. Vasseur, F. Grasset, E. Duguet, J. Mater. Chem. 14, 2161 (2004).

[7] P. Gould, Materials Today 2, 36 (2004). 
[8] K.S. Novoselov, A.K. Geim, S.V. Morozov, D. Jiang, M.I. Katsnelson, I.V. Grigorieva, S.V. Dubonos, A.A. Firsov, Nature 438, 197 (2005).

[9] Y. Zhang, Y.-W. Tan, H.L. Stormer, P. Kim, Nature 438, 201 (2005).

[10] S. Cudziło, M. Bystrzejewski, H. Lange, A. Huczko, Carbon 43, 1778 (2005).

[11] M. Bystrzejewski, H. Lange, A. Huczko, J. High Temp. Mater. Processes 9, 237 (2005).

[12] M. Bystrzejewski, A. Huczko, H. Lange, Sensors \& Actuators B 109, 81 (2005).

[13] M. Bystrzejewski, S. Cudziło, A. Huczko, H. Lange, J. Alloys Comp. 423, 74 (2006).

[14] M. Bystrzejewski, A. Huczko, H. Lange, S. Cudziło, W. Kiciński, Diamond Relat. Mater. 16, 225 (2007).

[15] M. Bystrzejewski, A. Huczko, H. Lange, P. Baranowski, Gervais Soucy, German Cota-Sanchez, J. Szczytko, A. Twardowski, Nanotechnology 18, 145608 (2007).

[16] M. Bystrzejewski, H. Lange, A. Huczko, Fullerenes, Nanotubes and Carbon Nanostructures 15, 167 (2007).

[17] E. Sasioglu, I. Galanakis, L.M. Sandratskii, P. Bruno, J. Phys., Condens. Matter 17, 3915 (2005).

[18] M. Megens, M. Prins, J. Magn. Magn. Mater. 293, 702 (2005). 